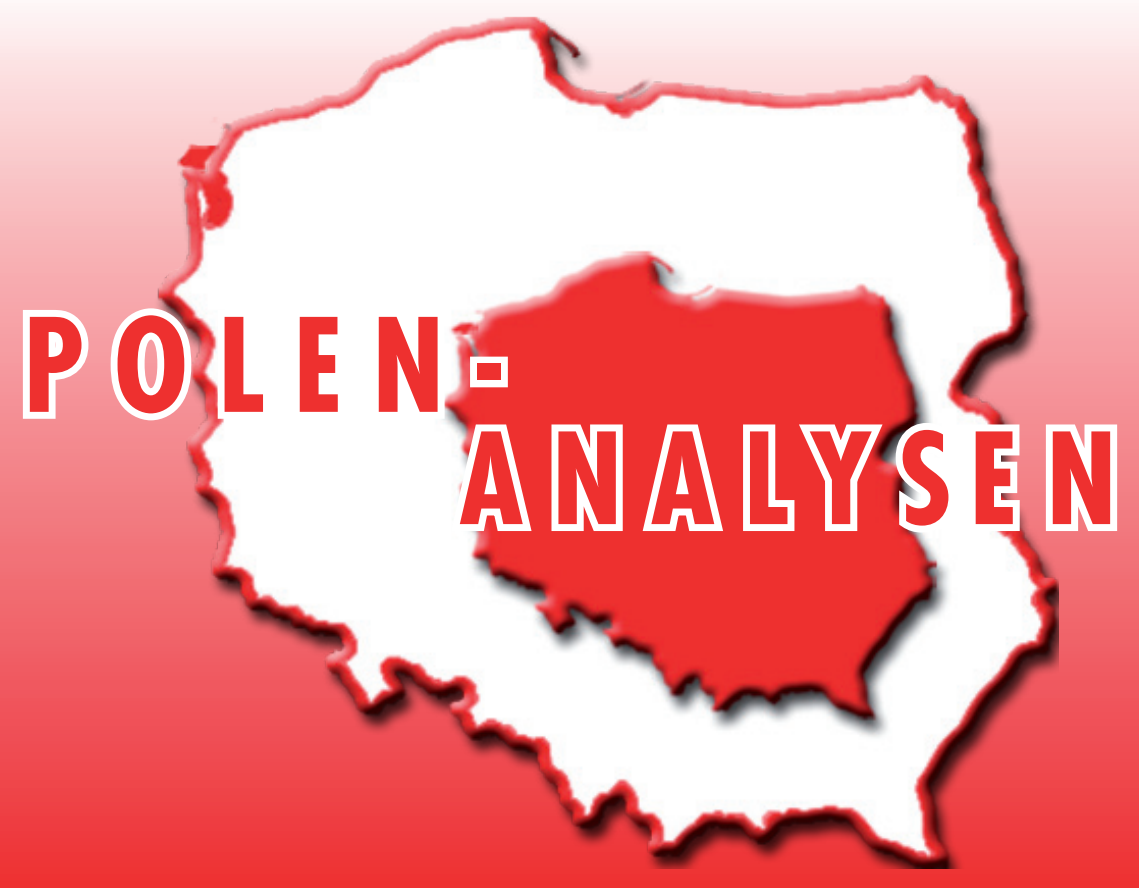

www.laender-analysen.de/polen

\title{
NACH DEN PARLAMENTSWAHLEN 2011
}

ANALYSE

Polen nach den Parlamentswahlen 2011

Janusz A. Majcherek, Krakau

- TABELLEN UND GRAFIKEN ZUM TEXT

Wahlergebnisse und Wahlbeteiligung (2011 und 2007)

6

- CHRONIK

Vom 4. bis zum 17. Oktober 2011 


\title{
Polen nach den Parlamentswahlen 2011
}

\author{
Janusz A. Majcherek, Krakau
}

\section{Zusammenfassung}

Der Sieg der Bürgerplattform (PO) bei den Parlamentswahlen ist beispiellos, kommt aber nicht überraschend. Quelle des Erfolgs und der Dominanz der PO in der polnischen Politik ist die Herausbildung einer neuen, jungen Mittelschicht, die zur festen gesellschaftlichen Basis dieser liberal-konservativen Partei geworden ist. Das Ergebnis des Wahlduells zwischen den beiden wichtigsten Parteien PO und PiS ist fast eine Neuauflage von vor vier Jahren. Es bestätigt sowohl die Stabilität des Parteiensystems als auch die Kluft der geistigweltanschaulichen Präferenzen innerhalb der polnischen Gesellschaft. Die größte Sensation dieser Wahlen, nämlich das plötzliche Auftauchen und hervorragende Abschneiden der Palikot-Bewegung ist ein Spiegelbild der wachsenden Forderungen nach Säkularisierung und Modernisierung. Das von PiS wieder ins Spiel gebrachte deutsche Thema hat der Partei offensichtlich geschadet. Das Wahlergebnis kann als Aufforderung zu einem gemäßigt reformorientierten Kurs im Inneren und zur Fortsetzung des integrationsfreundlichen europapolitischen Kurs von Ministerpräsident Donald Tusk verstanden werden.

$\mathrm{D}$ er Sieg der Bürgerplattform (PO) bei den Parlamentswahlen ist beispiellos, kommt aber nicht überraschend. Außergewöhnlich an ihm ist, dass eine Regierungspartei vom Wähler beauftragt wurde, auch die nächsten vier Jahre weiter zu regieren, was es seit 1989 in Polen nicht gegeben hat. Das ist jedoch keine Überraschung, denn die $P O$ gewinnt seit fünf Jahren alle Wahlen auf den verschiedensten Ebenen (die vorherigen Parlamentswahlen, zweimal die Kommunalwahlen, die Wahlen zum EU-Parlament und die Präsidentschaftswahlen). In derselben Zeit führte sie auch in fast allen Umfragen und ihren Spitzenpolitikern wurde das größte Vertrauen entgegengebracht.

\section{Ein Bedürfnis nach Kontinuität und Stabilität ...}

Quelle des Erfolgs und der Dominanz der PO in der polnischen Politik ist die Herausbildung einer neuen, jungen Mittelschicht im Zuge der Wirtschaftstransformation, die zur festen gesellschaftlichen Basis dieser liberal-konservativen Partei geworden ist. Die PO hat die absolute Mehrheit unter jungen Stadtbewohnern, die die Schule mindestens mit der mittleren Reife abgeschlossen haben und von denen es immer mehr gibt. Die stärker werdende Mittelschicht erwartet von der Politik die Gewährleistung stabiler Entwicklungsbedingungen, und die Bürgerplattform erfüllt diese Erwartungen. Dies ist besonders wichtig in einer Zeit globaler ökonomischer Erschütterungen und starker Krisentendenzen. Unter Ministerpräsident Donald Tusk und seiner Regierung schaffte es Polen, der Rezession zu entgehen, und blieb selbst in der tiefsten Krise des Wirtschafts- und Finanzsystems auf Erfolgskurs. Das ist ein zusätzlicher und wichtiger Grund für die Unterstützung der Wähler.
Als Garant der Stabilität war die $P O$ konkurrenzlos, denn die wichtigste Oppositionspartei rief zum entschiedenen Bruch mit der bisherigen Politik und zu tiefgreifenden, ja radikalen Veränderungen auf. Wie diese aussehen sollten, konnten sich die meisten Wähler leicht vorstellen, denn die von Jarosław Kaczyński geführte Partei Recht und Gerechtigkeit (PiS) hatte bereits in den Jahren 2005-2007 regiert, was im Gedächtnis geblieben war als eine Zeit der vom Zaun gebrochenen Konflikte, des Chaos und der aufgepeitschten Emotionen, die mit dem Scheitern der damaligen Regierung und der vorzeitigen Beendigung der Legislaturperiode ihr Ende fand. Die Angst vor einem Rückfall in jene Praktiken der Störung, ja Infragestellung der institutionellen Ordnung, der Spaltung der Gesellschaft und der Anheizung von Konflikten innerhalb Polens wie auch mit dessen Nachbarn (besonders mit Deutschland und Russland) mobilisierte die Wählerschaft der $P O$ maßgeblich - trotz gewisser Enttäuschungen (die nur teilweise etwas mit der Krise zu tun hatten) über die von ihrer Regierung in den letzten Jahren betriebenen Politik. Das betrifft insbesondere die Unzulänglichkeiten beim Ausbau der Infrastruktur, aber auch die zu risikoscheue und opportunistische Steuer- und Sozialpolitik, die zu einem Anstieg der Staatsverschuldung führte. Wirtschaftsexperten verlangten von der Regierung einhellig Reformen im Renten- und Sozialsystem, aber der Ministerpräsident verzichtete mit stoischer Ruhe auf jegliche schnellen Veränderungen. In seiner zweiten Regierungszeit werden einige davon nicht mehr zu vermeiden sein. Dies ergibt sich aus der Notwendigkeit, die Beitrittsbedingungen zur Eurozone zu erfüllen (Reduzierung des Haushaltsdefizits und der Inflation), sowie aus Anforderungen des polnischen Rechts (so verbietet die polnische Verfassung beispielsweise eine Staatsverschul- 
dung von mehr als $60 \%$ des jährlichen BIP, während sich das gegenwärtige Haushaltsdefizit dieser Grenze gefährlich nähert). Vor allem geht es um die Einschränkung der zahlreichen und für den Haushalt kostspieligen Rentenprivilegien für einige Berufsgruppen sowie um den weit verbreiteten Missbrauch von Steuererleichterungen.

Am schwierigsten wird die Verringerung der unzähligen und üppigen Subventionen für die Landwirtschaft, denn über sie wacht die Polnische Bauernpartei (Polskie Stronnictwo Ludowe - PSL), Koalitionspartner der Bürgerplattform in der vergangenen Legislaturperiode, den diese auch in der jetzt beginnenden benötigen wird (zur absoluten Parlamentsmehrheit von 231 Stimmen fehlen der PO 24). Eine Wiederauflage der bisherigen Koalition von $P O$ und $P S L$ ist ein zusätzlicher Stabilisator der polnischen Politik, mit ihr droht aber auch das Einfrieren einiger geforderter und für das Land notwendiger Reformen, die Partikularinteressen gefährden. Der immer noch riesige Anteil der Landbevölkerung (zirka ein Drittel der Polen) sowie der in der Landwirtschaft Beschäftigten (zirka ein Fünftel aller Arbeitnehmer, bei einem Anteil von $3 \%$ dieses Sektors am polnischen BIP) stellt ein ernstes sozioökonomisches Problem dar. Und die PSL ist nicht bereit, sich ihm zu stellen, könnte dies doch ihre Wählerschaft verschrecken.

Das Ergebnis des Wahlduells zwischen den beiden wichtigsten Parteien PO und PiS ist fast eine Neuauflage von vor vier Jahren (39 \% zu $29 \%$ gegenüber $41 \%$ zu $32 \%$ im Jahr 2007). Dies zeigt sowohl die Stabilität des Parteiensystems als auch die Kluft der geistig-weltanschaulichen Präferenzen innerhalb der polnischen Gesellschaft. PiS ist eine Partei mit nationalkatholischem Profil, die eine traditionalistische und konservative Wählerschaft repräsentiert. Für sie stimmen hauptsächlich Einwohner von Dörfern und Kleinstädten, die unter dem Einfluss der katholischen Kirche stehen und ein Anspruchsdenken gegenüber dem Staat haben, von dem sie eine paternalistische Politik erwarten. Die zahlenmäßige Stärke dieses Wählerpotentials ist rückläufig (PiS erhielt einige Hunderttausend Stimmen weniger als bei den vorigen Wahlen), und die Serie von sechs Wahlniederlagen hintereinander schwächt den Zusammenhalt und die Entschlossenheit dieser Partei. Nur vorübergehenden Aufwind brachte ihr die Flugzeugkatastrophe von Smolensk (Russland) im April 2010, bei der der polnische Staatspräsident Lech Kaczyński, der Zwillingsbruder des PiS-Vorsitzenden, zusammen mit vielen anderen Vertretern dieser Partei starb; ihre Aktivisten und Anhänger benutzten die Tragödie dazu, zahlreiche patriotische und Trauerkundgebungen zu veranstalten und der Regierung eine unzureichende Erfüllung ihrer Verpflichtungen vorzuwerfen (und auch ein zu enges Zusammenwirken mit den russischen Behörden bei der Vorbereitung des Unglücksflugs zum Ort des Gedenkens an den Tod der 1940 von der sowjetischen Geheimpolizei ermordeten polnischen Offiziere sowie bei der Aufklärung des Flugzeugabsturzes). Die ostentative Form dieser patriotischen Trauerrituale mit ihrer starken Betonung religiöser Symbolik (vor dem Präsidentenpalast wurde ein Kreuz aufgestellt, um dessen Beseitigung später ein langer und erbitterter Kampf geführt wurde) konsolidierte die Anhängerschaft von PiS, rief aber in anderen Teilen der Gesellschaft Unwillen, Abscheu und Widerspruch hervor.

\section{... und nach Wandel}

Das ist eine der Ursachen für die größte Sensation dieser Wahlen, nämlich das plötzliche Auftauchen und hervorragende Abschneiden der ad hoc gegründeten PalikotBewegung (Ruch Palikota), die mit ihrem Ergebnis von $10 \%$ zur drittstärksten Kraft im Sejm wurde. Janusz Palikot, nach dem die neue Partei benannt wurde, ist ein ehemaliger einflussreicher Politiker der $P O$, ein 46-jähriger exzentrischer und skandalumwobener Millionär (der in der Alkoholbranche reich geworden ist) und studierter Philosoph, der seit einiger Zeit immer gewagtere und provokativere Meinungen zu Fragen gesellschaftlicher Sitten und Gebräuche äußerte. Da er dafür in seiner gemäßigten und eher konservativen Mutterpartei weder Verständnis noch Resonanz fand, verließ er sie und gründete (mit seinem eigenen Geld) eine Bewegung für die sozio-kulturelle Rebellion. Zu seinem Markenzeichen wurde der Antiklerikalismus und zu seiner Hauptforderung die Säkularisierung des Staates. Rasch fand er breite Unterstützung in jüngeren, vor allem studentischen Kreisen (unter den 18- bis 25-jährigen Wählern lagen die Palikot-Bewegung und PiS gleichauf mit zirka $23 \%$ ).

Die Palikot-Bewegung ist eine dieser kurzlebigen und sonderbaren Protestparteien, die von Zeit zu Zeit in den demokratischen Ländern auftauchen (so die Partei der Komiker in Island, Liste Pim Fortuyn in den Niederlanden oder die Piratenpartei, die kürzlich bei den Wahlen zum Berliner Abgeordnetenhaus erfolgreich war). Nur teilweise kann überraschen, dass ihr Impetus von Rebellion und Protest sich gegen die katholische Kirche, ihre Rolle in Staat und Gesellschaft sowie gegen ihre Doktrin richtet. In einem so katholischen Land wie Polen, wo die katholische Kirche fast Teil des Establishments ist, ist die antikirchliche, antiklerikale Spitze des Protestes gegen das Establishment fast selbstverständlich. Die sich in Polen vollziehenden Modernisierungsprozesse haben einen schon seit langem beobachteten Rückgang des Einflusses der Kirche auf die polnische Gesellschaft und ein immer geringer werdendes Engagement der Bürger in ihren Strukturen zur Folge, vor allem aber - was im 
politischen Kontext besonders wichtig ist - eine wachsende Abneigung gegen den Einfluss kirchlicher Institutionen auf nichtreligiöse Bereiche (zwei Drittel der Polen meinen, der Einfluss der Kirche auf die Politik in Polen sei zu groß, 80 \% wünschen nicht, dass sie die Entscheidung staatlicher Funktionsträger beeinflusst, und $85 \%$ sprechen ihr das Recht ab, den Bürgern Wahlempfehlungen zu geben). Lauter werden auch die Forderungen, den säkularen Charakter des Staates, eine laizistische Bildung und die weltanschauliche Neutralität im politischen Bereich zu wahren. Der plötzliche Erfolg der antiklerikalen Palikot-Bewegung spiegelt diese Tendenzen wider, mit denen Politiker der großen Parteien immer mehr rechnen müssen, allen voran die Regierungspartei (denn an sie, als für die Politik des Staates Verantwortliche, werden diese Erwartungen und Forderungen gerichtet werden).

Aber die Palikot-Bewegung steht für einen noch breiter angelegten gesellschaftlich-kulturellen Protest, der auch Losungen und Forderungen wie die nach der Einführung von eingetragenen - auch homosexuellen - Lebenspartnerschaften, der Liberalisierung des Abtreibungsrechts, der Kostenübernahme für künstliche Befruchtung (In-vitro-Fertilisation) und sogar der Legalisierung von Marihuana umfasst. Auf den Listen dieser Partei ins Parlament gewählt wurden beispielsweise der Aktivist einer Homosexuellenorganisation, eine Transsexuelle, die vor kurzem noch ein Mann war (wohl gegenwärtig die Einzige in einem Parlament weltweit), und der Vertreter einer Bewegung für die Legalisierung weicher Drogen. Das verändert die polnische politische Landschaft oder macht sie zumindest bunter, und dies wahrscheinlich unumkehrbar. Die Rolle der Kirche im polnischen öffentlichen Leben und besonders in der Politik ist schwächer geworden und wird sich weiter abschwächen.

\section{Die traditionelle Linke im Aus}

Nicht imstande, diese Entwicklungen und Tendenzen vorherzusehen oder sie politisch zu nutzen, war die traditionelle Linke, die bei den Wahlen eine beispiellose Niederlage erlitt. Ihre Anführer und Kandidaten versuchten, Forderungen nach Säkularisierung und Modernisierung mit der nach einem breiten Ausbau des Sozialsystems zu verbinden. Es zeigt sich jedoch, dass ein solches Angebot im gesellschaftlichen Nirwana landet. Milieus und Gruppen mit Anspruchshaltung an den Staat, von dessen Fürsorge und Sozialpolitik sie abhängig sind, die ausgeschlossen oder sozial oder kulturell benachteiligt sind, wählen eher die nationalkatholische $P i S$, suchen Trost in der Religion und Rückhalt bei der Kirche. Die liberal und säkular eingestellten Emanzipierten gehören wiederum in ihrer Mehrheit zur aktuellen und poten- tiellen Mittelschicht und sind für soziale Versprechungen nicht empfänglich (gerade bei ihnen konnte die Palikot-Bewegung punkten). Außerdem konnte die PO einen Teil der linken Funktionäre und Wähler auf ihre Seite ziehen. Das waren diejenigen, die ernsthaft eine Rückkehr von PiS an die Macht befürchteten und lieber für die einzige Partei stimmten, die das verhindern konnte, sowie Anhänger derjenigen linken Politiker, denen die PO Plätze auf ihren Wahllisten versprochen hatte. Ein spektakuläres Beispiel dafür war der Erfolg des ehemaligen linken Abgeordneten Bartosz Arłukowicz der nach seinem Wechsel zur PO im Wahlkreis Stettin für sie antrat, wo er den dort kandidierenden (und von dort stammenden) Vorsitzenden der Demokratischen Linksallianz (Sojusz Lewicy Demokratycznej - SLD), Grzegorz Napieralski, klar besiegte. Letzterer kündigte nach dieser politischen und persönlichen Niederlage folgerichtig den Rücktritt von seinem Amt an (obwohl er mit erst 37 Jahren eine große Hoffnung der Linken war).

Die Zukunft der Linken in Polen ist die große Unbekannte. Die postkommunistische Klientel, die traditionell die Linke wählt, ist in Auflösung begriffen. Für die zahlenmäßig wachsende Mittelschicht hat die Linke nichts anzubieten, was mit der $P O$ konkurrieren könnte. Die rebellisch, emanzipatorisch und libertär eingestellte Jugend hat (für wie lange, weiß man nicht) eine andere politische Vertretung gefunden. Die Linke muss beginnen, sich selbst zu erneuern. Vorläufig sind jedoch keine neuen Ideen und Gedanken in Sicht.

\section{Noch einmal das deutsche Thema}

In der Endphase des Wahlkampfs kam unerwartet das deutsche Thema ins Spiel. Jarosław Kaczyński gab vor den Wahlen sein Buch heraus, in dem aufmerksame Leser einen Absatz über Angela Merkel fanden. Dort deutete der PiS-Vorsitzende an, sie würde ihre Position irgendwelchen geheimnisvollen Machenschaften verdanken und insgeheim sei die Unterordnung Polens ihr Bestreben. Es kam zum Skandal, der Kaczyński und seiner Partei deutlich schadete. Damit zeigte sich, dass der Versuch, die antideutsche Karte zu spielen, nicht nur Wählerstimmen in Polen kostet, sondern auch dessen Urheber kompromittiert.

Anlässlich dieses Skandals und als Reaktion auf die dubiosen Anschuldigungen Kaczyńskis beeilte man sich, die große Bedeutung der deutsch-polnischen Beziehungen hervorzuheben, an die Verdienste Deutschlands und auch persönlich von Bundeskanzlerin Merkel für Polen zu erinnern. Die Bedeutung der Zusammenarbeit wurde betont, die vielversprechenden Zukunftsperspektiven für die beiderseitigen Beziehungen dargelegt. Donald Tusk, der weiter die Regierung führen wird, ist bekannt für seine engen und guten Beziehungen zu Angela Mer- 
kel. Sein Wahlsieg und die Verlängerung seiner Amtszeit als polnischer Regierungschef stehen für die Fortsetzung der deutsch-polnischen Annäherung und Vertiefung der Zusammenarbeit. Wenn nichts Unvorhergesehenes geschieht, werden die deutsch-polnischen Beziehungen in den kommenden Jahren immer besser werden.

An den Wahlen nahm traditionsgemäß auch eine Vertretung der deutschen Minderheit teil, per Wahlgesetz von der 5-Prozent-Hürde befreit, die zur Erlangung von Abgeordnetenmandaten erforderlich ist. Sie errang jedoch lediglich ein Mandat (in ihrer besten Zeit, zu Beginn der 1990er Jahre, war sie mit sieben Abgeordneten im Sejm vertreten, seitdem nimmt ihre Unterstützung systematisch ab, was belegt, dass ihre Mitglieder für gesamtpolnische Parteien stimmen, vorwiegend für die $P O)$. Traditionell unterstützen die Abgeordneten der deutschen Minderheit die jeweils gewählte Regierung.

Neben den Wahlen zum Sejm fanden auch die Wahlen zur zweiten Kammer des polnischen Parlaments statt, dem 100 Mitglieder umfassenden Senat. Sie verdienen besondere Beachtung wegen der erstmalig eingeführten Einmandatwahlkreise. Obwohl es Versuche gab, diese Chance für unabhängige Kandidaten zu nutzen, spiegelten die Ergebnisse die Dominanz der beiden großen Parteien wider, mit klarem Vorsprung für die $P O$, die 63 Senatoren stellen wird, gegenüber 31 von $P i S$, zwei PSL-Senatoren und vier Unabhängigen. Der Senat spielt allerdings im polnischen politischen System nur eine untergeordnete Rolle, die mit der des deutschen Bundesrates nicht vergleichbar ist. Er könnte wichtig werden, wenn die Opposition im Sejm die Mehrheit in der zweiten Kammer erreichen würde und damit Gesetzesvorhaben der Regierung blockieren könnte. Angesichts der klaren Dominanz der $P O$ droht die Gefahr einer solchen Sackgasse in der Legislative jedoch nicht.

\section{Optionen des alten und neuen Regierungschefs}

Da Polen gegenwärtig (zweites Halbjahr 2011) die turnusmäßige Ratspräsidentschaft in der Europäischen Union innehat, will Ministerpräsident Tusk den Kabinettswechsel so durchführen, dass dieser die Arbeit der Regierungsadministration so wenig wie möglich stört. Die Bildung der neuen Regierung wird also ohne Eile, störungsfrei und ohne abrupte Wendungen, unter maximaler Ausnutzung der gesetzlich dafür vorgesehenen Zeit vonstattengehen. Das neue Kabinett soll organisatorisch und teilweise auch personell umbesetzt werden. Zu den Ministern, die mit Sicherheit in ihren bisherigen Ämtern bleiben, gehört Außenminister Radosław Sikorski. Gewisse Probleme bereiten dem Ministerpräsidenten die vom Koalitionspartner PSL designierten Minister. Unter ihnen war die bisher umstrittene Sozi- alministerin (oft im Konflikt mit dem Finanzminister, da sie eigene, nicht abgesprochene Ideen verkündete), die eine Wahlniederlage hinnehmen musste.

Der Ministerpräsident ist theoretisch in einer guten Position für die Verhandlungen mit dem Koalitionspartner über die Zusammensetzung der Regierung, denn hypothetisch könnte er alternative Möglichkeiten der Verständigung mit anderen Fraktionen suchen. In der Praxis stellt dies jedoch keine echte Perspektive dar. Ein Zusammengehen mit der SLD würde ein Bündnis mit einer durch ihre Wahlniederlage kompromittierten Partei bedeuten, die ohne gesellschaftlichen Auftrag und schwächer als die PSL ist (die parlamentarische Mehrheit wäre dann noch geringer). Die Aufnahme einer Zusammenarbeit mit der Palikot-Bewegung, also einer vor kurzem erfolgten Abspaltung von der eigenen Partei, würde bedeuten, dass man den Bruch von vor einem Jahr als Fehler eingesteht. So bleibt nur die Neuauflage der bisherigen Koalition mit der PSL. Der Ministerpräsident kann jedoch auf eine gewisse Unterstützung von Seiten Palikots zählen, hat dieser doch seine Unterstützung all derjenigen Regierungsentscheidungen angekündigt, die mit den Forderungen seiner Bewegung übereinstimmen. Diese zu erfüllen, wird allerdings schwierig sein, denn ein Teil von ihnen ist unrealistisch (Legalisierung von Marihuana), ein anderer Teil könnte aber im Falle ihrer Durchsetzung Widerstand beim konservativen Flügel der $P O$ hervorrufen, ja sogar eine Spaltung der Partei herbeiführen. So bleibt als einzig reale Möglichkeit die Koalition mit der PSL, also die Fortsetzung der bisherigen Politik, mit einigen geringen Korrekturen.

Ministerpräsident Tusk setzt mehr auf Kontinuität (bestätigt durch die gute Bewertung der bisherigen Regierungspolitik durch die Wähler), doch in seiner Partei und Fraktion gibt es Befürworter gewichtiger Korrekturen und auch grundlegender Reformen. Diese haben die makroökonomischen Notwendigkeiten und steuerpolitischen Gefahren im Blick, die die Umstrukturierung der öffentlichen Finanzen erforderlich machen, einschließlich einer Kürzung und Neuordnung der Ausgaben. Auf mutigere Reformen drängt angeblich auch der Staatspräsident, der aus der $P O$ kommende Bronisław Komorowski. Da Ministerpräsident Tusk angekündigt hat, er werde sich in einer weiteren Legislaturperiode nicht mehr um die Position des Regierungschefs bemühen, muss er nicht politisch taktieren und kann sich eine risikofreudigere Politik mutigerer Reformen erlauben. Noch ist keineswegs absehbar, welche dieser Tendenzen die Oberhand gewinnen wird. Zum Teil hängt das auch von der Entwicklung der wirtschaftlichen Situation in Europa und der Welt ab, die ebenfalls nicht vorhersehbar ist.

Übersetzung aus dem Polnischen: Ulrich Heiße Informationen über den Autor finden Sie aufdernächsten Seite 
Über den Autor

Janusz A. Majcherek ist Professor am Institut für Philosophie und Soziologie der Pädagogischen Hochschule in Krakau. Seit 1989 publiziert er Analysen und Kommentare zu politischen und gesellschaftlichen Themen in der polnischen Presse und äußert seine Standpunkte in Rundfunk und Fernsehen. Er arbeitet mit dem deutsch-polnischen Magazin "Dialog« zusammen und hat mehrere Beiträge im "Jahrbuch Polen« des Deutschen Polen-Instituts Darmstadt sowie in der Zeitschrift »OST-WEST. Europäische Perspektiven« veröffentlicht.

\section{Wahlergebnis und Wahlbeteiligung}

Grafik 1: Die Sitzverteilung im Sejm 2011

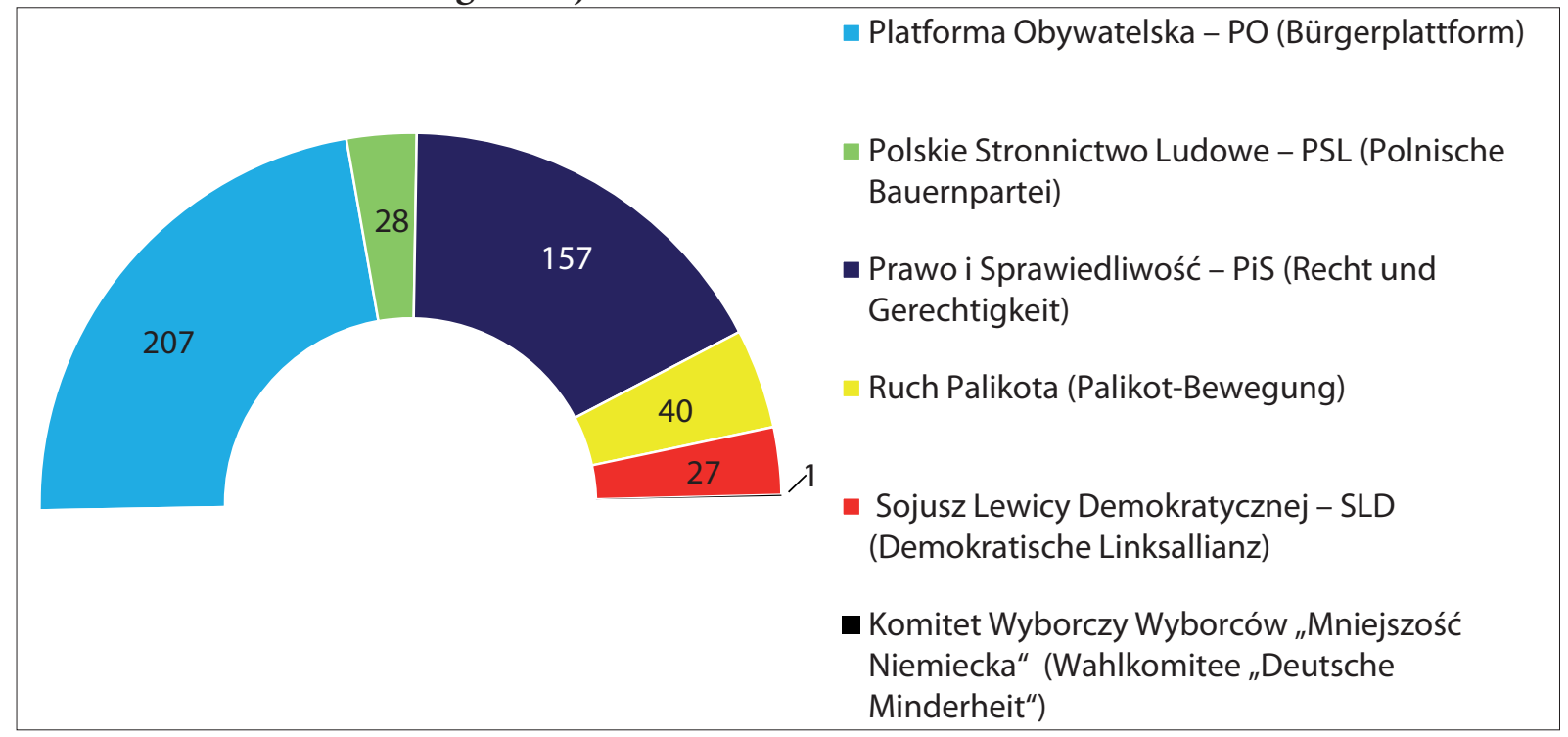

Quelle: Państwowa Komisja Wyborcza [Staatliche Wablkommission], www.pkw.gov.pl

Grafik 2: Die Sitzverteilung im Sejm 2007

\begin{tabular}{|c|c|} 
— Platforma Obywatelska - PO (Bürgerplattform) \\
- Polskie Stronnictwo Ludowe - PSL (Polnische \\
Bauernpartei) \\
- Prawo i Sprawiedliwość - PiS (Recht und \\
Gerechtigkeit) \\
Lewica i Demokraci - LiD (Wahlbündnis Linke \\
und Demokraten) \\
- Komitet Wyborczy Wyborców "Mniejszość \\
Niemiecka“ (Wahlkomitee "Deutsche \\
Minderheit")
\end{tabular}

Quelle: Państwowa Komisja Wyborcza [Staatliche Wablkommission], www.pkw.gov.pl 
Grafik 3: Die Sitzverteilung im Senat 2011

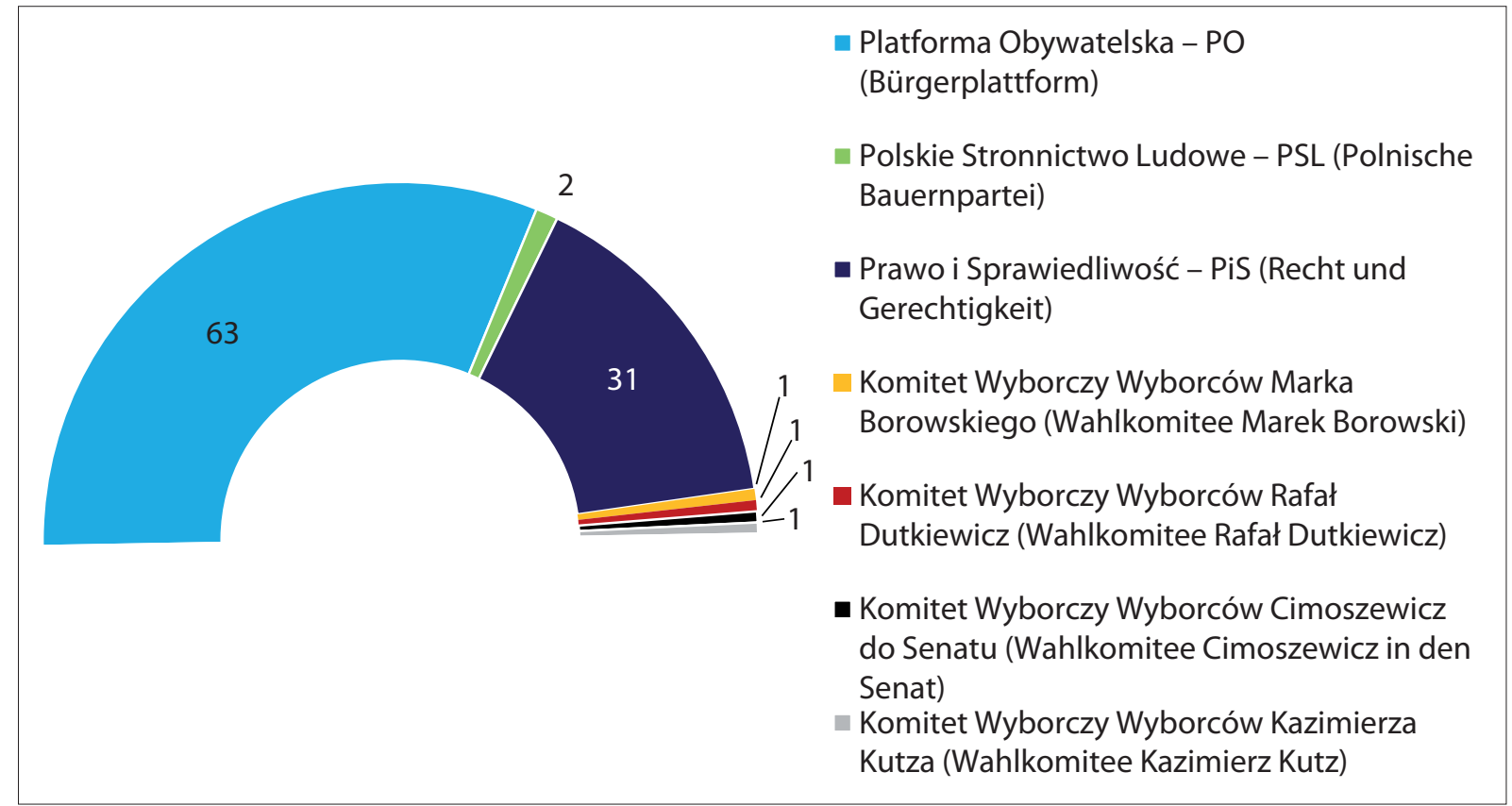

Quelle: Państwowa Komisja Wyborcza [Staatliche Wahlkommission], www.pkw.gov.pl

Grafik 4: Die Sitzverteilung im Senat 2007

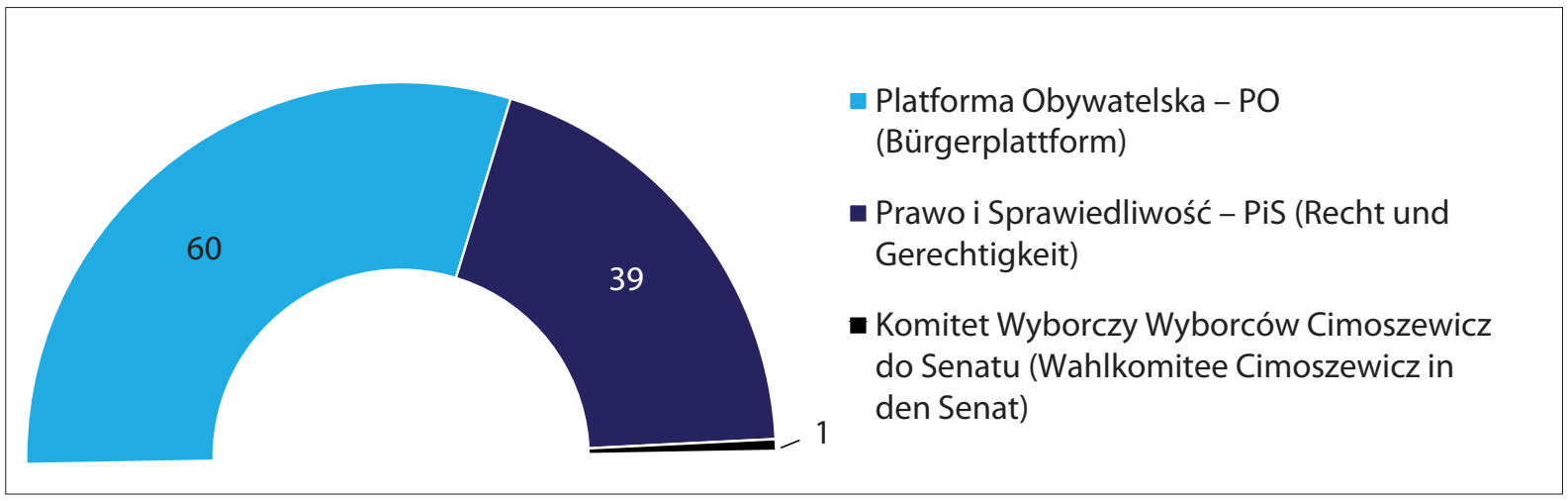

Quelle: Państwowa Komisja Wyborcza [Staatliche Wahlkommission], www.pkw.gov.pl 
Grafik 5: Stimmenanteile bei der Sejmwahl am 9.10.2011

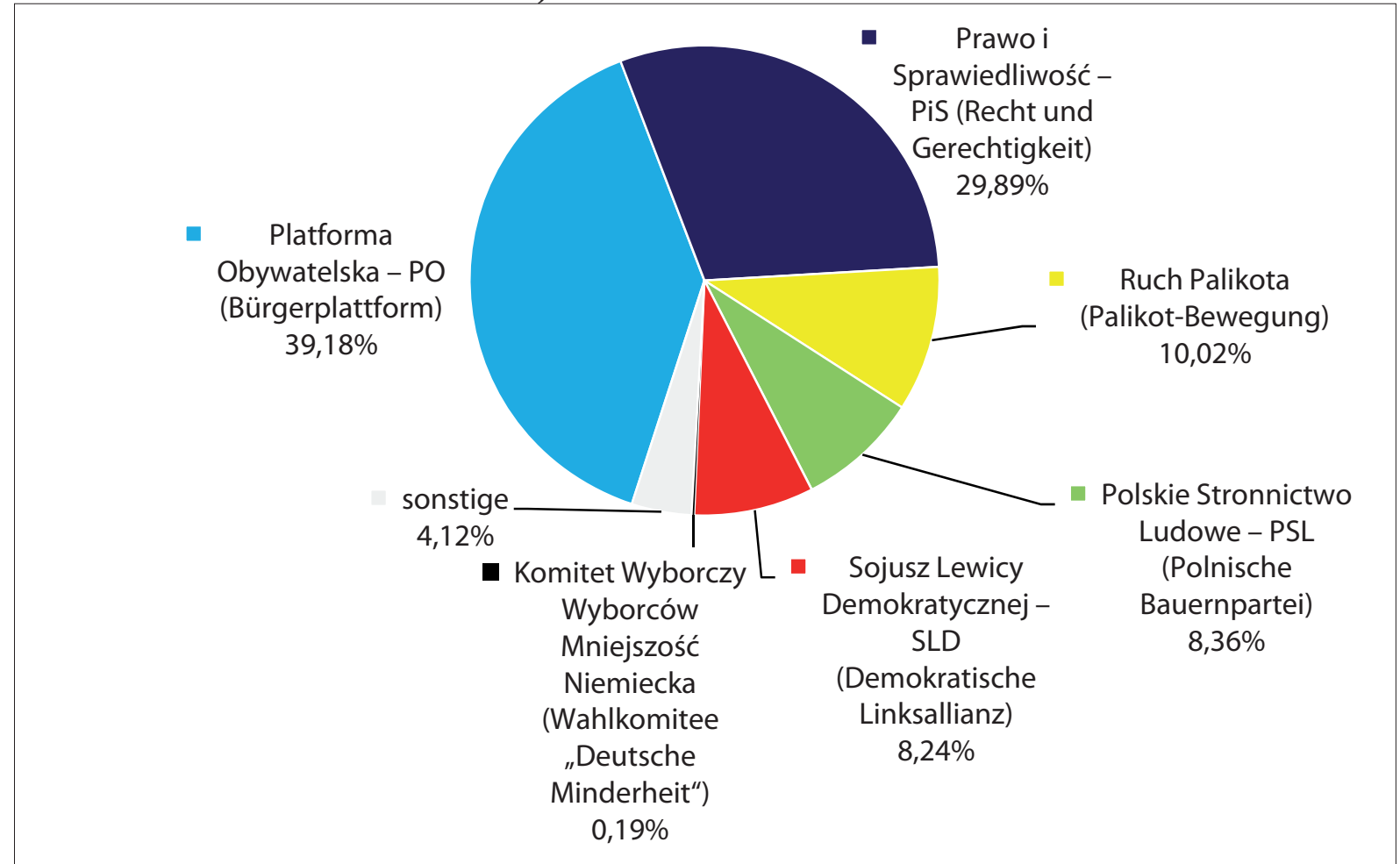

Quelle: Państwowa Komisja Wyborcza [Staatliche Wahlkommission], www.pkw.gov.pl

Grafik 6: Stimmenanteile bei der Sejmwahl am 21.10.2007

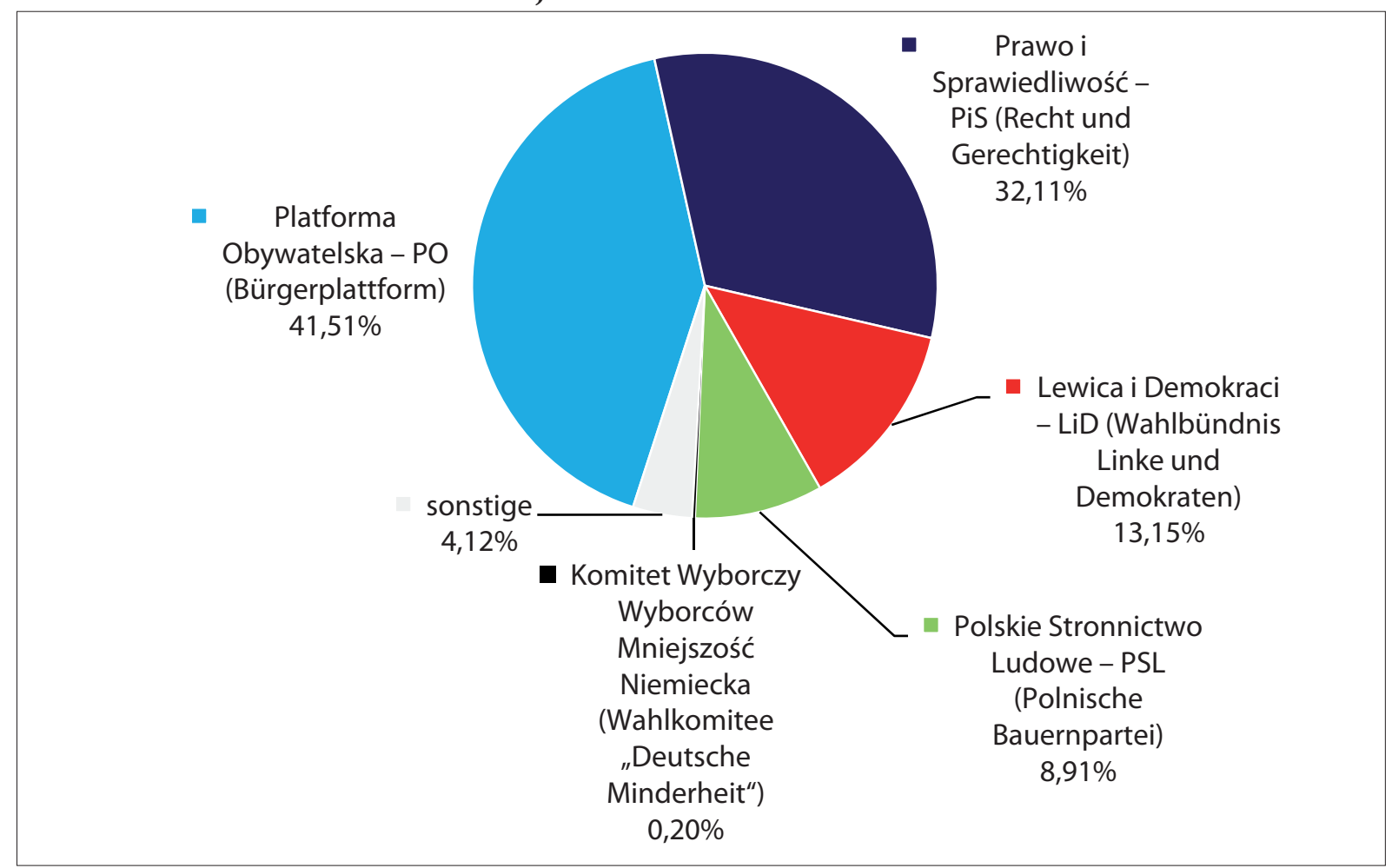

Quelle: Państwowa Komisja Wyborcza [Staatliche Wablkommission], www.pkw.gov.pl 
Tabelle 1: Die Verteilung der Abgeordneten im Sejm nach Alter und Geschlecht (Parlamentswahlen 2011)

\begin{tabular}{|l|c|c|c|c|c|c|}
\hline \multicolumn{1}{|c|}{ Alter } & $\begin{array}{c}\text { Anzahl der } \\
\text { Abgeordneten }\end{array}$ & $\begin{array}{c}\text { Abgeordnete } \\
\%\end{array}$ & $\begin{array}{c}\text { Anzahl der } \\
\text { Frauen }\end{array}$ & Frauen \% & $\begin{array}{c}\text { Anzahl der } \\
\text { Männer }\end{array}$ & Männer \% \\
\hline 21-29 Jahre & 10 & 2,17 & 1 & 0,22 & 9 & 1,96 \\
30-39 Jahre & 86 & 18,70 & 12 & 2,61 & 74 & 16,09 \\
\hline 40-49 Jahre & 135 & 29,35 & 33 & 7,17 & 102 & 22,17 \\
\hline 50-59 Jahre & 160 & 34,78 & 40 & 8,70 & 120 & 26,09 \\
\hline 60-69 Jahre & 66 & 14,35 & 23 & 5,00 & 43 & 9,35 \\
\hline 70 Jahre + & 3 & 0,65 & 1 & 0,22 & 2 & 0,43 \\
\hline insgesamt & 460 & 100 & 110 & 23,91 & 350 & 76,09 \\
\hline
\end{tabular}

Quelle: Państwowa Komisja Wyborcza [Staatliche Wahlkommission], www.pkw.gov.pl

Tabelle 2: Die Verteilung der Senatoren im Senat nach Alter und Geschlecht (Parlamentswahlen 2011)

\begin{tabular}{|c|c|c|c|c|c|c|}
\hline \multicolumn{1}{|c|}{ Alter } & $\begin{array}{c}\text { Anzahl der } \\
\text { Senatoren }\end{array}$ & Senatoren \% & $\begin{array}{c}\text { Anzahl der } \\
\text { Frauen }\end{array}$ & Frauen \% & $\begin{array}{c}\text { Anzahl der } \\
\text { Männer }\end{array}$ & Männer \% \\
\hline 30-39 Jahre & 4 & 4 & 1 & 1 & 3 & 3 \\
\hline 40-49 Jahre & 19 & 19 & 2 & 2 & 17 & 17 \\
\hline 50-59 Jahre & 45 & 45 & 5 & 5 & 40 & 40 \\
\hline 60-69 Jahre & 27 & 27 & 4 & 4 & 23 & 23 \\
\hline 70 Jahre + & 5 & 5 & 1 & 1 & 4 & 4 \\
\hline insgesamt & 100 & 100 & 13 & 13 & 87 & 87 \\
\hline
\end{tabular}

Quelle: Państwowa Komisja Wyborcza [Staatliche Wohlkommission], www.pkw.gov.pl

Grafik 7: Wahlbeteiligung an den Sejmwahlen am 9.10.2011 und am 21.10.2007 nach Städten über 250.000 Einwohner

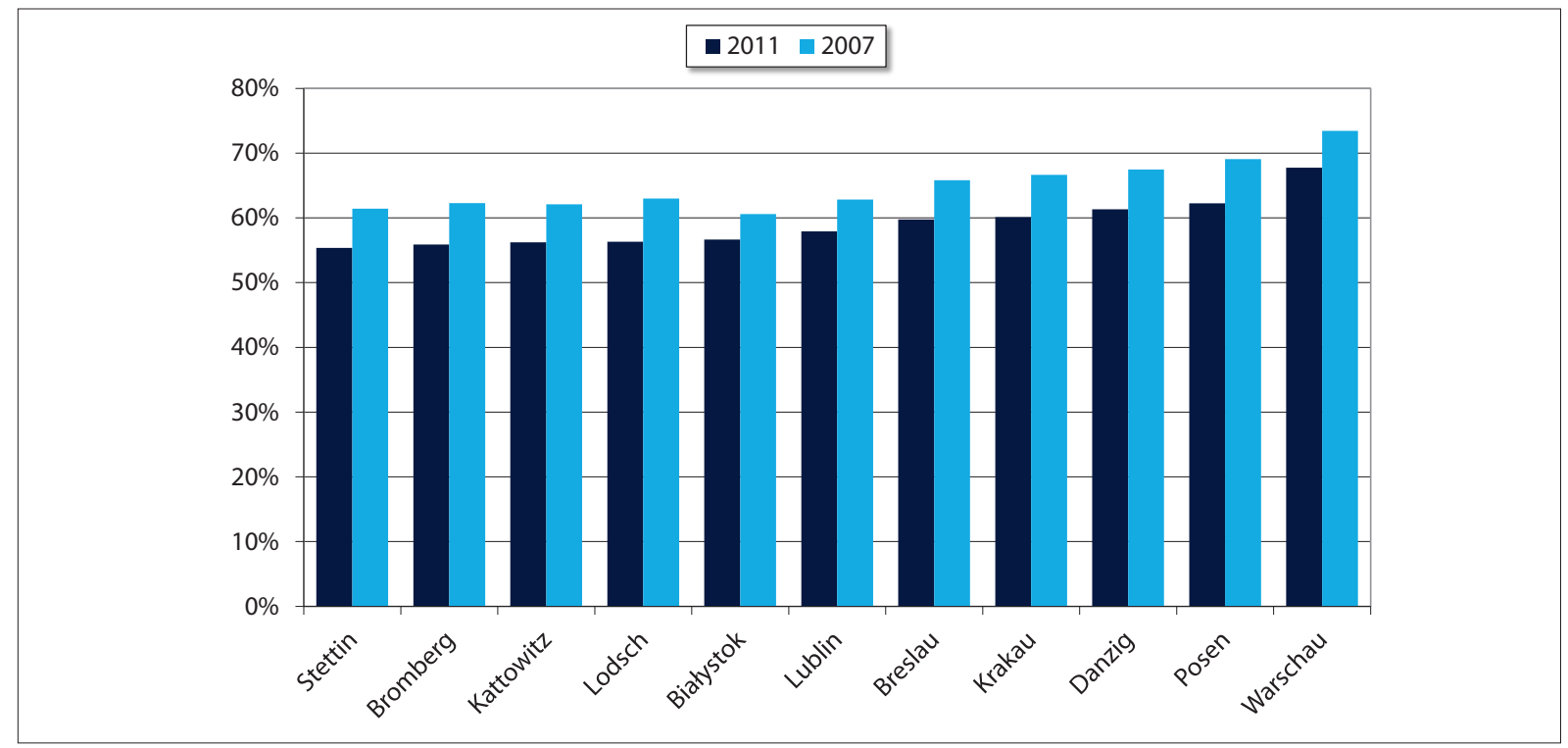

Quelle: Państwowa Komisja Wyborcza [Staatliche Wahlkommission], www.pkw.gov.pl 
Tabelle 3: Wahlbeteiligung an den Sejmwahlen am 9.10.2011 und am 21.10.2007 nach Städten über 250.000 Einwohner

\begin{tabular}{|l|c|c|}
\hline & 2011 & 2007 \\
\hline Białystok & $56,66 \%$ & $60,60 \%$ \\
\hline Breslau (Wrocław) & $59,76 \%$ & $65,81 \%$ \\
\hline Bromberg (Bydgoszcz) & $55,89 \%$ & $62,27 \%$ \\
\hline Danzig (Gdańsk) & $61,32 \%$ & $67,47 \%$ \\
\hline Kattowitz (Katowice) & $56,32 \%$ & $62,09 \%$ \\
\hline Krakau (Kraków) & $60,12 \%$ & $66,66 \%$ \\
\hline Lodsch (Lódź) & $56,33 \%$ & $63,00 \%$ \\
\hline Lublin & $57,91 \%$ & $62,84 \%$ \\
\hline Posen (Poznań) & $62,26 \%$ & $69,08 \%$ \\
\hline Stettin (Szczecin) & $55,37 \%$ & $61,43 \%$ \\
\hline Warschau (Warszawa) & $67,75 \%$ & $73,44 \%$ \\
\hline
\end{tabular}

Quelle: Panstwowa Komisja Wyborcza [Staatliche Wablkommission], www.pkw.gov.pl

Grafik 8: Wahlbeteiligung an den Sejmwahlen in Polen (in \%)

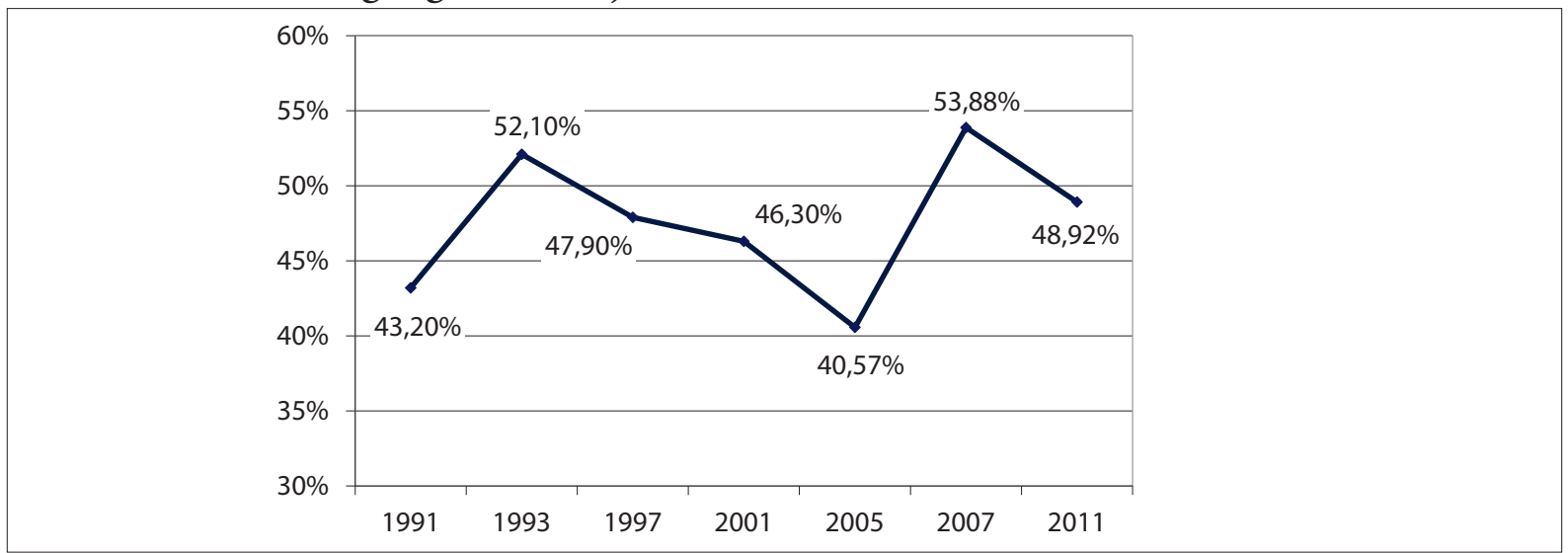

Quelle: Państwowa Komisja Wyborcza [Staatliche Wablkommission], www.pkw.gov.pl

\section{CHRONIK}

\section{Vom 4. bis zum 17. Oktober 2011}

\begin{tabular}{|l|l|}
\hline 04.10 .2011 & $\begin{array}{l}\text { In der letzten Wahlkampfphase spielt der Vorsitzende der Partei Recht und Gerechtigkeit (Prawo i Sprawiedli- } \\
\text { wość - PiS), Jarosław Kaczyński, offensiv die deutsche Karte aus. Es wird bekannt, dass er in seiner jüngst erschie- } \\
\text { nenen politischen Publikation und einem Interview behauptet, hinter der Wahl von Angela Merkel zur Bundes- } \\
\text { kanzlerin hätten »dunkle Mächte" gestanden. Die PiS-Politikerin und ehemalige Außenministerin Anna Fotyga } \\
\text { beschuldigt in einer Fernsehdebatte deutsche Architekten und Baufirmen, die am Bau der Fußballstadien betei- } \\
\text { ligt waren, der Untreue. Ein anonymer Informant, der die Heizungsleistung des Warschauer Nationalstadions als } \\
\text { zu überdimensioniert kritisierte, erwies sich als Fotygas Ehemann. }\end{array}$ \\
\hline 05.10 .2011 & $\begin{array}{l}\text { Ministerpräsident Donald Tusk kündigt eine Kabinettsumbildung und Änderungen der Ressortstruktur im Falle } \\
\text { eines Wahlsieges an. So soll der Infrastrukturbereich vom Innenministerium abgetrennt und als Transportminis- } \\
\text { terium in den nächsten Jahren die Entwicklung des polnischen Bahnwesens fördern. Ein Ministerium für Digitali- } \\
\text { sierung soll die Wende bei der flächendeckenden Versorgung mit digitalen Technologien herbeiführen. Ebenso soll } \\
\text { ein Zentrum für Strategische Planung eingerichtet werden, das beim Ministerpräsidenten direkt angesiedelt wäre. }\end{array}$ \\
\hline
\end{tabular}




\begin{tabular}{|c|c|}
\hline 05.10 .2011 & $\begin{array}{l}\text { Die fünf ehemaligen Außenminister Władysław Bartoszewski, Włodzimierz Cimoszewicz, Andrzej Olechowski, } \\
\text { Dariusz Rosati und Adam Rotfeld bekunden in einem offenen Brief ihren »scharfen Protest« gegen die jüngs- } \\
\text { ten Äußerungen des Parteivorsitzenden von Recht und Gerechtigkeit (Prawo i Sprawiedliwość - PiS), Jarosław } \\
\text { Kaczyński, über die polnisch-deutschen Beziehungen und verwahren sich gegen dessen Auslassungen über Bun- } \\
\text { deskanzlerin Angela Merkel: ßesondere Unruhe weckt die Unterstellung, die Wahl in das Amt der Bundeskanz- } \\
\text { lerin von Frau Angela Merkel sei „kein reiner Zufall gewesen«, heißt es in dem Brief. Kaczyński hatte am Vortag } \\
\text { vor der Presse gesagt, er sehe keine Gründe, sich bei Angela Merkel zu entschuldigen. }\end{array}$ \\
\hline 09.10 .2011 & $\begin{array}{l}\text { Die Wahlen zu den beiden Kammern des Parlaments, Sejm und Senat, finden in einer ruhigen Atmosphäre statt. } \\
\text { Unmittelbar nach Schließung der Wahllokale um } 21 \text { Uhr wird aufgrund von exit polls der Wahlsieg der regieren- } \\
\text { den Bürgerplattform (Platforma Obywatelska - PO) vorausgesagt und ein Ergebnis für den Koalitionspartner Pol- } \\
\text { nische Bauernpartei (Polskie Stronnictwo Ludowe - PSL), das an das Ergebnis von } 2007 \text { heranreicht. Der Vor- } \\
\text { sprung der PO gegenüber Recht und Gerechtigkeit (Prawo i Sprawiedliwość - PiS) beträgt ungefähr } 10 \text { Prozent. } \\
\text { Die Palikot-Bewegung (Ruch Palikota), eine links-libertäre Protestpartei, zieht überraschend als drittstärkste Partei } \\
\text { ins Parlament ein. Die Demokratische Linksallianz (Sojusz Lewicy Demokratycznej - SLD) wird stark geschwächt. }\end{array}$ \\
\hline 10.10.2011 & Der Dichter und Dramatiker Tadeusz Różewicz feiert seinen 90. Geburtstag. \\
\hline 10.10.2011 & $\begin{array}{l}\text { Als Reaktion auf das katastrophale Wahlergebnis kündigt der Vorsitzende der Demokratischen Linksallianz (Sojusz } \\
\text { Lewicy Demokratycznej -SLD), Grzegorz Napieralski, einen außerordentlichen Parteitag an, auf dem er nicht } \\
\text { mehr zum Vorsitzenden der Partei kandidieren wird. }\end{array}$ \\
\hline 11.10.2011 & $\begin{array}{l}\text { Die Staatliche Wahlkommission (Państwowa Komisja Wyborcza) gibt das offizielle Wahlergebnis bekannt: Bür- } \\
\text { gerplattform (Platforma Obywatelska - PO) 39,18\% (207 Mandate), Recht und Gerechtigkeit (Prawo i Sprawie- } \\
\text { dliwość - PiS) 29,89\% (157), Palikot-Bewegung (Ruch Palikota) 10,02\% (40), Polnische Bauernpartei (Polskie } \\
\text { Stronnictwo Ludowe - PSL) 8,36\% (28), Demokratische Linksallianz (Sojusz Lewicy Demokratycznej - SLD) } \\
\text { 8,24\% (27). Das Wahlkomitee der deutschen Minderheit (Komitet Wyborczy Wyborców Mniejszość Niemiecka) } \\
\text { erhält } 1 \text { Mandat. Die Wahlbeteiligung betrug 48,92\%. }\end{array}$ \\
\hline 11.10.2011 & $\begin{array}{l}\text { Staatpräsident Bronisław Komorowski kündigt an, Donald Tusk als Chef der stärksten Partei im Parlament (Bür- } \\
\text { gerplattform/Platforma Obywatelska - PO) wieder mit der Regierungsbildung zu betrauen. Es gibt erste Sondie- } \\
\text { rungsgespräche zur Bildung einer Koalition zwischen der PO und der Polnischen Bauernpartei (Polskie Stron- } \\
\text { nictwo Ludowe - PSL), aber die PO schließt auch eine Zusammenarbeit mit der Palikot-Bewegung (Ruch Pali- } \\
\text { kota) nicht aus. }\end{array}$ \\
\hline 13.10.2011 & $\begin{array}{l}\text { ent Donald Tusk kündigt die Neubesetzung des Amts des Sejmmarschalls an. Neue Parlaments- } \\
1 \text { die bisherige Gesundheitsministerin Ewa Kopacz von der Bürgerplattform (Platforma Obywatel- } \\
\text { den, der bisherige Sejmmarschall Grzegorz Schetyna (PO) soll ein Regierungsressort übernehmen. }\end{array}$ \\
\hline 13.10 .2011 & $\begin{array}{l}\text { er designierte Ministerpräsident Donald Tusk will zunächst Koalitionsgespräche mit der Polnischen Bauernpar- } \\
\text { i (Polskie Stronnictwo - PSL) führen. Der Vorsitzende der PSL, Waldemar Pawlak, beansprucht weiterhin drei } \\
\text { inisterien für seine Partei. }\end{array}$ \\
\hline 13.10 .2011 & $\begin{array}{l}\text { nusz Palikot fordert öffentlich die Entfernung des Kreuzes aus dem Plenarsaal des Sejm. Er bietet der Regierung } \\
\text { usammenarbeit bei Reformen an, die auf Zuspruch in seiner Partei (Palikot-Bewegung/Ruch Palikota) stoßen. }\end{array}$ \\
\hline 14.10 .2011 & $\begin{array}{l}\text { taatspräsident Bronisław Komorowski kündigt die Beauftragung von Donald Tusk mit der Regierungsbildung auf } \\
\text { er konstituierenden Sejmsitzung (7. Legislaturperiode) am 8.11. an. Am selben Tag werde er eine Rede im Sejm } \\
\text { alten. Außerdem kündigt er die konstituierende Sitzung des Senats (8. Legislaturperiode) für denselben Tag an. }\end{array}$ \\
\hline 15.10.2011 & $\begin{array}{l}\text { Im Zusammenhang mit den Protesten in vielen europäischen und außereuropäischen Ländern u. a. gegen die prak- } \\
\text { tizierte Finanzpolitik und innenpolitische Missstände in den jeweiligen Staaten sowie das Verhalten der Finanz- } \\
\text { branche findet in Warschau ein Protestmarsch statt. Die Polizei spricht von zirka 150, die Organisatoren von zirka } \\
800 \text { Teilnehmern. }\end{array}$ \\
\hline 16.10.2011 & $\begin{array}{l}\text { Im Auftrag des Statistischen Instituts der Katholischen Kirche (Instytut Statystyki Kościoła Katolickiego) werden an } \\
\text { diesem Sonntag die Besucher der katholischen Messen sowie die Teilnehmer an der Heiligen Kommunion gezählt. }\end{array}$ \\
\hline 17.10.2011 & $\begin{array}{l}\text { Der Europaabgeordnete und ehemalige Vorsitzende der Demokratischen Linksallianz (Sojusz Lewicy Demokra- } \\
\text { tycznej - SLD), Wojciech Olejniczak, spricht sich dafür aus, dass die Wahl der neuen SLD-Führung in einem } \\
\text { Referendum durchgeführt wird, an dem sich alle Sympathisanten der Linken beteiligen können sollten. Vorbild } \\
\text { sei für ihn die Kür des Präsidentschaftskandidaten der französischen Linken. }\end{array}$ \\
\hline
\end{tabular}


Die Polen-Analysen erscheinen zweimal monatlich als E-Mail-Dienst. Sie werden gemeinsam vom Deutschen PolenInstitut Darmstadt, der Bremer Forschungsstelle Osteuropa und der Deutschen Gesellschaft für Osteuropakunde herausgegeben.

Ein Archiv der Polen-Analysen finden Sie im Internet unter www.laender-analysen.de/polen

Kostenloses Abonnement unter http://www.deutsches-polen-institut.de/Newsletter/subscribe.php

Diese Analysen finden Sie online als Lizenzausgabe auf

bpb.de

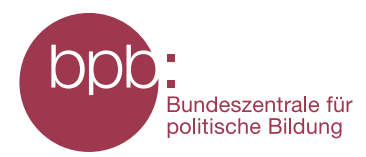

\section{Deutsches Polen-Institut Darmstadt}

Das Deutsche Polen-Institut Darmstadt (DPI) ist ein Forschungs-, Informations-, und Veranstaltungszentrum für polnische Kultur, Geschichte, Politik, Gesellschaft und die deutsch-polnischen Beziehungen, die sich im Kontext der europäischen Integration entwickeln. Das seit März 1980 aktive und bis 1997 von Gründungsdirektor Karl Dedecius geleitete Institut ist eine Gemeinschaftsgründung der Stadt Darmstadt, der Länder Hessen und Rheinland-Pfalz sowie des Bundes. 1987 wurden die Kultusminister der Länder und 2011 das Auswärtige Amt weitere institutionelle Träger. Einen wesentlichen Beitrag zur Verwirklichung der Institutsziele leisten private Stiftungen. Das DPI hat satzungsgemäß die Aufgabe, durch seine Arbeit zur Vertiefung der gegenseitigen Kenntnisse des kulturellen, geistigen und gesellschaftlichen Lebens von Polen und Deutschen beizutragen.

Ziel der Vermittlertätigkeit des DPI ist es, »die zu interessieren, auf die es politisch, wirtschaftlich, gesellschaftlich und kulturell im deutsch-polnischen Verhältnis ankommt« (Leitlinien 1997). Es geht um die Entscheider und Multiplikatoren in Politik, Kultur, Bildung, Verwaltung, Medien und Wirtschaft und, wesentlich stärker ausgeprägt als bisher, um das Hineinwirken in Wissenschaft, Forschung und Bildung.

Derzeit bemüht sich das DPI in Kooperation mit den verstreuten Orten wissenschaftlicher Polen-Kompetenz an deutschen Hochschulen und Forschungsinstituten verstärkt darum, ausgehend von einer Bestandsaufnahme deutscher Polen-Forschung Ort wissenschaftlicher Forschung und verbindendes, vernetzendes und kooperierendes Zentrum zu werden. Ausgangspunkt der Neuausrichtung ist die kaum mehr kontrollierbare Dynamik des Rückbaus der Ressourcen der wissenschaftlichen Polen-Kompetenz in den unterschiedlichen Disziplinen. Mit der knapp 60.000 Bände zählenden multidisziplinären Fachbibliothek für Polen, die eine einzigartige Sammlung polnischer Literatur in der Originalsprache und in deutscher Übersetzung umfasst, ist das DPI bereits ein geschätzter Ort der Recherche und des wissenschaftlichen Arbeitens. (www.deutsches-polen-institut.de)

\section{Forschungsstelle Osteuropa an der Universität Bremen (www.forschungsstelle.uni-bremen.de)}

1982 gegründet, widmet sich die Forschungsstelle Osteuropa an der Universität Bremen der interdisziplinären Analyse der Länder Ost- und Ostmitteleuropas in Zeitgeschichte und Gegenwart. Der Forschungsschwerpunkt liegt dabei auf der Rolle von "Dissens und Konsens«, von Opposition und Zivilgesellschaft in ihrem historischen, politischen, gesellschaftlichen und kulturellen Kontext. Die Forschungsstelle besitzt in ihrem Archiv eine einzigartige Sammlung alternativer Kulturgüter und unabhängiger Texte aus den ehemaligen sozialistischen Ländern. Darunter befindet sich auch eine umfangreiche Sammlung des "Zweiten Umlaufs», die das Schrifttum und Dokumente unabhängiger Initiativen und gesellschaftlicher Gruppen in Polen aus der Zeit von 1976 bis zum Umbruch umfasst. Hinzu kommt eine umfangreiche Bibliothek mit wissenschaftlicher Literatur. Mit Archiv, Bibliothek und zwei wissenschaftlichen Abteilungen ist die Forschungsstelle auch eine Anlaufstelle sowohl für Gastwissenschaftler als auch für die interessierte Öffentlichkeit.

Eine der Hauptaufgaben der Forschungsstelle ist die Information der interessierten Öffentlichkeit. Dazu gehören unter anderem regelmäßige E-Mail-Informationsdienste für Politik, Wirtschaft, Zivilgesellschaft und Medien. 